\title{
Ulysse Guinand (1810-1885), un géographe suisse romand influent jadis, oublié aujourd'hui
}

\section{Bernard Huber, Genève}

\section{Eléments biographiques}

Influent géographe suisse du XIXe siècle, principal auteur romand de manuels scolaires de géographie de cette époque, aujourd'hui presque complètement oublié, U. Guinand ne fait l'objet d'aucun travail de recherche approfondi. Aussi estimons-nous intéressant de consacrer le présent texte à ce pédagogue.

U. Guinand naît aux Brenets, dans la principauté de Neuchâtel. Petit-fils d'un célèbre opticien, P.-L. Guinand, sa formation scolaire s'effectue à La Chaux-deFonds, Neuchâtel et Lausanne. En 1830, il est nommé maître d'histoire et de géographie à Neuchâtel. Profondément républicain, il s'oppose, par le biais d'une activité politique et journalistique débordante, à l'aristocratie neuchâteloise. Désertant, un jour, son poste d'enseignant pour se rendre aux Brenets, soupçonné d'y mener des activités révolutionnaires, le géographe est arrêté le 18 décembre 1831 et jeté en prison. Il y croupit seize jours. Dans sa séance du 27 décembre 1831, le Conseil général décide de déclarer vacant son poste.

U. Guinand se rend à Lausanne. Dans un premier temps, il est nommé professeur extraordinaire à l'Académie de cette ville; dans un second, il obtient un poste à l'Ecole normale du chef-lieu vaudois (TAUXE 1922).

Les détails de son entrée à l'Ecole normale nous sont fournis par un document manuscrit intitulé Procès-verbaux des séances du Comité de l'Ecole normale (ANonyme 1833-1834). Nous y apprenons qu'en 1833, alors que L.-F.-F. GAUTHEY, directeur, est responsable de l'enseignement de la géographie, les ouvrages utilisés sont ceux de F. DE Rougemont. Cette même année, alors qu'U. Guinand n'est pas encore nommé à l'Ecole normale, il fait aux élèves de cette dernière une offre de cours de géographie gratuits. L'offre est acceptée. Très suivis, ils sont d'ailleurs dispensés non seulement aux futurs enseignants, mais également aux enseignants eux-mêmes, désireux de se perfectionner. U. Guinand sait se faire apprécier. Il le sait si bien que le Comité de l'Ecole normale va désirer l'employer régulièrement. En haut lieu, son mérite est reconnu. Certains vont même jusqu'à dire qu'il serait avantageux pour le canton de Vaud de conserver ce jeune géographe. Le 15 août 1834, il est ainsi nommé maître de géographie dans cet établissement. Il remplace à ce poste le directeur. U. Guinand y enseigne jusqu'en 1845, année de la Révolution vaudoise. Il est en effet remercié cette année-là. Nous n'avons malheureusement pas réussi à déterminer les circonstances exactes de son départ. Il travaille, dès lors, dans des pensionnats. Aucun renseignement relatif à cette étape de sa vie ne nous est connu.

Darbyste, U. Guinand exerce une intense activité religieuse dans son canton d'adoption, qui ne sera pas sans influencer sa pensée géographique.

Il s'éteint à Lausanne.

\section{U. Guinand dans le contexte scientifique de son époque. Entre géographie de tradition française et géographie d'obédience germanique: un abîme}

Il sied de replacer U. Guinand dans le contexte scientifique du début du XIXe siècle (cf. Schlup 1991, Robert 1909, Knapp 1886, Marti-Henneberg 1986). Pour lui, il y a un «avant» et un «après» C. Ritrer. En 1834, il écrit:

«Il y a quarante ans à peine que la géographie était encore complètement abandonnée à l'empire de la routine. La plus haute prétention d'une description de la terre était, on est tenté de le croire, de recouvrir les aspérités de la statistique et de la topographie, de phrases nombreuses et académiques.» (Guinand 1834:5)

Il reconnaît toutefois qu'au siècle précédent, des tentatives ont été faites pour tirer de son marasme cette science. Et U. Guinand de citer P. Buache, L.-A. Lacroix et J.-B. Bory de SAINT-VInCEnt. Mais, constate-t-il, la géographie reste ce qu'elle est, une aride nomenclature, une sèche description de faits isolés. Les liens qui unissent les divers objets géographiques ne sont pas encore mis en valeur. Les ouvrages pédagogiques de D.-F. De Merveilleux (1694) et, surtout, de F.-S. Ostervald (1773) sont les principaux exemples suisses romands de cette géographie préritterienne. Ils utilisent la méthode catéchétique, faite de questions et de réponses, alors très en vogue.

U. Guinand disserte longuement sur les différences entre la géographie de tradition française et celle d'obédience germanique. Il constate que la première est, au début du XIXe siècle, en état de léthargie. Son représentant le plus important est un Danois établi à Paris, K. Malte-Brun (1775-1826).

U. Guinand critique très vivement l'œuvre de ce dernier. Pour lui, K. Malte-Brun est le principal porteparole, en France, de la géographie de type analytique. L'un des principaux partisans de la philosophie analytique, dans ce pays, est E. BONNOT DE CONDILLAC. L'analyse, nous dit U. Guinand, peut trouver 
des applications légitimes; il en est ainsi de l'anatomie qui, convenablement employée par C. Cuvier, fait de cette discipline une véritable science. Il en est également ainsi, estime cet auteur, de la chimie et de la géologie. Le grand tort de certains scientifiques et philosophes du XVIIIe siècle, pense-t-il, c'est d'avoir exagéré sa portée. Elle n'est pas applicable à tout. Qu'en est-il de la géographie?

"C'eût été chose merveilleuse que cette impulsion se fût arrêtée devant les études géographiques. Bien au contraire, elles devaient être les premières à s'y soumettre. Qu'y a-t-il en effet de plus important en géographie que de connaître et d'examiner les faits? Ne semble-t-il pas que tout doive se borner à les énumérer, à les peser, à les constater, afin d'établir leur certitude, leur caractère, leur valeur absolue et relative? Or c'est l'analyse qui donne tout cela; ses procédés étaient donc éminemment applicables à la géographie. L'analyse avait mission de disséquer le globe et elle l'a fait.» (Guinand 1834:9)

C'est, pense le Neuchâtelois, en Angleterre et en France que les idées de la philosophie analytique se sont propagées le plus dans le domaine qui nous intéresse. Les pays nouvellement découverts sont examinés à la loupe. Chaque aspect (orographie, hydrographie, populations, langues, religions, etc.) est analysé dans les plus petits détails. En revanche, aucune vision d'ensemble. Les liens entre les divers aspects d'une région ne sont pas mis en évidence. Or, pour U. Guinand, l'analyse des faits géographiques ne suffit pas. En s'y bornant, on n'obtient qu'une sèche nomenclature. De telles notions (noms, chiffres, divisions de toute sorte, etc.), quoique nécessaires, ne forment pas une science. Usant de la métaphore, il dit:

«Sous cette croûte qu'il faut percer, circule une vie admirable. Il faut la saisir, l'étudier, la comprendre. Vous possédez à merveille toute la masse des faits géographiques? C'est bien, mais c'est peu. Rendez la vie à ce cadavre immense, étendu dans votre mémoire.» (Guinand 1834: 11)

La Terre est un tout pour K. Malte-Brun, admet U. Guinand. Mais, constate-t-il, c'est une unité dont il n'a pas encore saisi l'intelligence. Ce tout n'est composé que de parties disposées sans motif les unes à côté des autres. K. Malte-Brun, qui se proclame lui-même le «coryphée de la géographie», n'a fait de cette discipline, estime le Neuchâtelois, qu'une récréation, un amusement de l'esprit. K. Malte-Brun, pense-t-il en substance, est bien en retard par rapport à l'évolution de cette science.

A la critique, notre auteur ajoute parfois le sarcasme: K. Malte-Brun ne serait que le pire des routiniers (sic). Son œuvre ne serait que le développement des abrégés les plus arides (sic) et les plus repoussants (sic). Cette sécheresse, concède-t-il, est atténuée par un style attrayant, voire brillant.

U. Guinand, laissant de côté sa véhémence, reonnaît pourtant au géographe danois, outre son style, un autre mérite, plus «géographique». En effet, K. MALTE-Brun, contrairement à ses pairs exerçant en France, tient compte de l'action de l'homme sur la nature, comme de la réaction de la nature sur l'homme.

Le Neuchâtelois pense qu'en France,

«... on n'a fait qu'entrevoir encore la science: on n'a point soulevé le voile et pénétré dans le sanctuaire.... les savants allemands, ont il y a 15 à 20 années créé la science géographique et RITTER, à Berlin, enseigne en pleine université, ce qu'on cherche encore inutilement à Paris." (Guinand, cité par RogivUe \& SECRETAN 1832: IV)

Nous avons lu maints textes, français pour la plupart, dans lesquels était signalée cette torpeur qui caractérise la géographie française au début du XIXe siècle. Il nous paraît intéressant, à cet égard, de prendre connaissance des propos tenus par un étudiant neuchâtelois d'U. Guinand qui a également suivi des cours de géographie à la Sorbonne. Ce témoignage met en évidence l'abîme qui sépare un enseignement préritterien, celui de la Sorbonne, d'un enseignement postritterien qui commence à se répandre dans le monde germanique et qu' $U$. Guinand pense incarner en Suisse romande. Cet étudiant suit les cours d'U. Guinand entre juin et novembre 1830. Du journal qu'il tient dès son arrivée à Paris, le même mois, nous extrayons le passage suivant, qui date du 16 décembre:

«Leçon de BarbiÉ Du BocaGe (professeur de géographie). Neuchâtel, quelle petite ville vis-à-vis de Paris! Les professeurs de Neuchâtel, quels petits professeurs en comparaison de ceux de Paris! Le plan d'éducation de Neuchâtel, comme c'est borné, auprès des grands cours, des grandes leçons, de la fameuse Sorbonne, la lumière du monde! Il est vrai qu'à Neuchâtel, en sortant du collège, à seize, à dix-sept ans, nous savons fort bien notre géographie, ou, si nous ne la savons pas, c'est notre faute, et que j'ai vu à Paris deux étudiants de vingt à vingt-cinq ans se disputer sur ce que c'était que la mer Baltique, et la chercher inutilement sur la carte pendant près de cinq minutes, au bout desquelles, prenant pitié d'eux, j'ai bien voulu la leur indiquer. Et pourtant, Dieu sait si je suis fort en géographie! Il est vrai aussi que j'ai entendu quelques leçons de notre jeune professeur Guinand sur la géographie physique, les plus intéressantes qu'il soit possible d'entendre, et où je n’ai pas bâillé une seule fois; et je sors d’une leçon du fameux BarbiÉ du BocaGe où je me suis mortellement ennuyé, et où $\mathrm{j}$ 'ai appris qu'il y avait toutes sortes de mers: la mer Blanche, la mer Adriatique, la mer de Marmara, la mer Noire, la mer d'Azof, ce qui étonnait fort les étudiants de vingt-cinq ans et plus que j'avais à côté de moi et qui cherchaient avec empressement sur les petites cartes qu'on leur avait distribuées, se demandant à chaque mot: «Où est-ce que c'est ça? Qu'est-ce qu'il dit?». Il est vrai aussi que la faute n'était pas toute aux étudiants et que, quand M. Barbié du Bocage sautait de la mer de Guinée à la mer Caspienne, de la mer Rouge à la mer Bleue, de la mer Glaciale à la Terre de Feu, il y avait là de quoi déso- 
rienter de plus habiles qu'eux et donner du fil à retordre à d'autres qu'à des étudiants parisiens.» (GODET, F., cité par Godet, P. 1913: 50-51)

A l'instar du Neuchâtelois F. DE RougEmont, élève de C. RITTER à Berlin et premier propagateur de ses idées dans le monde francophone (Le Précis de géographie comparée, de F. DE Rougemont est, à cette époque (1831), le seul ouvrage écrit en français présentant la doctrine de C. RITTER dans son ensemble. Soulignons toutefois qu'il ne s'agit pas véritablement d'une traduction de l'Introduction à la géographie générale comparée du géographe allemand. Il ne faut pas perdre de vue le fait que la traduction partielle de cette œuvre que donnent E. Buret et E. Desor ne date que de 1836. P. Chaix confirme ce point de vue. Pour lui, les idées de C. RITTER ont bel et bien été introduites dans le monde francophone par F. DE Rougemont. De retour dans sa patrie, ce dernier a popularisé «...le feu sacré dérobé à l'autel de Berlin.» (CHAIX 1885:6). Dans son introduction à la traduction que donne $\mathrm{D}$. NiCOLAS-OBADIA de l'Introduction à la géographie générale comparée, G. Nicolas-OBadia, tout en signalant la présence d'étudiants suisses à Berlin, n'évoque pas la personnalité, essentielle à nos yeux, de F. DE RougeMONT. Il ne signale pas, non plus, la même origine neuchâteloise d'E. DESOR, l'un des deux traducteurs de 1836. Ce dernier était un Allemand d'origine française, naturalisé suisse (Neuchâtel) en 1859. La question de l'origine de ces deux personnalités qui, à première vue, peut paraître sans intérêt est, au contraire, importante. Pourquoi? Parce qu'elle met en évidence le caractère crucial que joue Neuchâtel relativement à la diffusion dans le monde francophone de la pensée ritterienne. Cette réalité s'explique aisément. Le canton de Neuchâtel, entré dans la Confédération suisse en 1815 , demeure concomitamment principauté prussienne jusqu'en 1848. Non seulement d'importants liens politiques sont tissés avec Berlin et ce, depuis longtemps, mais également nombre d'échanges d'ordre intellectuel sont établis avec la Prusse.), U. Guinand, adepte du grand géographe allemand, se targue d'être un pionnier dans le domaine de la diffusion de la pensée ritterienne. Il est conscient du rôle qu'il joue dans la transmission de cette dernière. Usant de propos quelque peu pontifiants et ampoulés, ne dit-il pas en effet:

«...l'auteur prie ses lecteurs, s'il en a, de ne pas perdre de vue que n'étant pas le créateur de la science, les idées qu'il expose sont, en très grande partie, celles de savans (sic) recommandables et surtout de RITTER, qu'il n'a pas cité assez souvent peut-être. A chacun son développement et sa doctrine. Celui qui le premier a essayé de professer la géographie comparée dans la Suisse française, laisse avec plaisir à d'autres le mérite de la science; il lui suffit de pouvoir être l'instrument de sa propagation.»(Guinand 1834:4)?

Nous verrons plus loin qu'entre l'expression sincère d'une volonté - propager la pensée ritterrienne - et sa traduction dans les faits, il y a un pas qu'U. Guinand peine à franchir.

\section{L'organisation de la Terre: un dessein d'essence divine}

Quittant le cadre strict du théâtre européen mais continuant à se pencher sur la question des diverses conceptions de la géographie, U. Guinand met en opposition celle, analytique, qu'a du monde la géographie française avec celle, hypersynthétique, de l'Inde. Ce faisant, il reprend une idée émise par C. RITTER.

Partant de l'idée qu'il est impossible que notre globe ne soit pas un objet organisé comme l'est le moindre animal, la moindre plante qui y vit, la géographie doit se fixer comme but de rechercher l'organisation du globe, comme le zoologue recherche l'organisation de l'animal et le botaniste, celle de la plante. L'animal, la plante, sont des œuvres de Dieu, des objets organisés. Le globe, par extrapolation, l'est aussi. Pour lui, il n'est pas pensable que la Terre ne soit qu'un agrégat de parties sans liaison entre elles. Derrière la perfection qu'elle représente, il y a nécessairement un projet d'ordre divin.

Cet auteur prend donc comme exemple de conception hypersynthétique du monde, le cas de la pensée brahmanique. Le brahmane, faisant abstraction des détails relatifs aux divers objets géographiques, fait de notre globe une véritable manifestation divine, d'une harmonie parfaite. Pour lui, tout est Dieu. La nature est l'expression de celui-ci comme l'est la Terre en général. Il a une vision d'ensemble du monde. Diviser ce dernier en objets géographiques revient à «découper» Dieu. La géographie est donc englobée dans la théologie. C'est, dit-il, dans les védas et les pouranas que nous trouvons les connaissances géographiques de l'Inde. Chacun des pouranas commence par un chapitre de géographie, ajoute-t-il. Transposant maintenant cette dichotomie orient/occident sur le théâtre européen, U. Guinand compare le peuple allemand au peuple indien. Pour lui, celui-là constitue en quelque sorte l'«orient» de notre continent.

Selon notre pédagogue, A. von Humboldt est le premier Européen à avoir attiré l'attention du monde scientifique occidental sur cette unité des faits géographiques. Cette idée est développée par C. RITTER qui «...a fait pour les continens (sic) ce que NEwTon a fait pour les mondes; il a trouvé le lien qui existe entr'eux (sic), leurs rapports réciproques, leur influence mutuelle. NEwTON a rattaché, par l'attraction, notre planète à d'autres corps célestes: il a trouvé la loi harmonieuse des mouvemens (sic) des mondes. Ritter a ramené l'unité entre les continens (sic), en saisissant la loi de leur développement.» (Guinand 1834: 19)

U. Guinand établit un autre parallèle intéressant entre la situation qui prévaut entre l'Asie et l'Europe d'une 
part et celle qui existe entre l'Allemagne et la France d'autre part: si l'Indien n'a pas de vision matérielle, pratique, de la science, l'Européen, en général, l'a. De même, la nature essentiellement spéculative des théories qui naissent en Allemagne ne revêt un caractère positif et pratique qu'en traversant le Rhin vers l'ouest, en direction de la France

«...puis, ainsi transformée, elle retourne dans sa patrie native, où elle apparaît presque comme un phénomène nouveau.» (GUinand 1834: 18)

Les lignes ci-dessus mettent en évidence le fait que si, pour l'auteur qui nous intéresse ici, chaque objet géographique peut être étudié pour lui-même, il convient avant tout de rechercher les liens qui l'unissent à d'autres. Ce postulat amène le géographe à se poser la question épistémologique de savoir où s'arrête exactement l'objet de la géographie. Est-ce aux limites de notre planète? Non, répond-il, car cette dernière n'est pas isolée dans l'univers. Elle subit les influences d'autres astres (la lune et son action dans le domaine des marées, pour ne prendre que cet exemple). Est-ce alors aux frontières extérieures du système solaire? Non! En effet, ce système est lui-même un point infime dans l'univers, imperceptible, subissant les influences d'astres ou de groupes d'astres d'un autre niveau. Ce nouveau centre d'action lui-même n'est pas immobile; il résulte du rôle que joue dans l'univers d'autres entités.

«Et quand, par une suite non interrompue de causes et d'effets, on aurait embrassé tout l'empire du fini, la science resterait encore vide de sens. Le fini n'est point sa propre cause à lui-même; sa cause est hors de lui. La plante la plus chétive comme l'harmonie sublime des mouvemens (sic) du ciel, la vie informe des végétaux comme les élans audacieux de l'intelligence humaine, tout atteste une cause suprême hors du fini, par laquelle seule le fini s'explique. Cette cause infinie, toute puissante, qui réunit en elle toutes les perfections...» (Guinand 1831:126),

c'est Dieu. U. Guinand rejoint donc C. RITTER, qui fait jouer à la Providence un rôle tout à fait important en matière d'explication de notre monde. Comme chez son maître, la dimension spirituelle du «tout», des «touts», est extrêmement marquée chez le Neuchâtelois. Les références à Dieu, au Créateur, sont innombrables dans ses textes. A propos de cette communion d'idées entre C. Ritrer et lui, cette citation, relative à la géographie, est significative:

«...son point d'appui, c'est la conscience des rapports de

Dieu au monde. Ce point d'appui n'est pas une vérité exclusive et particulière; il est l'ensemble de toutes les vérités; il est, pour parler avec Ritrer, du domaine de la foi. En effet, le matérialisme, qui repousse un créateur comme principe de tout, ne pouvait jamais arriver à la science de la terre.» (Guinand 1834: 30)

Dans la nature, rien n'est dû au hasard pense U. Guinand. Et ce, ajoute-t-il, quelle que soit l'échelle où nous nous situons. Quant à la science, elle n'explique pas tout, dit-il en d'autres termes.
Le Neuchâtelois distingue la cause première, Dieu, des causes secondes, l'homme et la nature. Les causes secondes ne permettent pas de comprendre tous les phénomènes observables à la surface du globe. Elles sont un moyen, utile et nécessaire, de parvenir à une meilleure interprétation du dessein de la cause première, suprême.

"Comprendre la nature, c'est connaître les faits et leurs rapports, c'est savoir en vertu de quelle loi tel phénomène a eu lieu et quelle cause a produit tel effet. Or, tout cela s'explique par des lois naturelles: mais en suivant cette route, on ne tarde pas à arriver à des vérités que l'on désigne quelquefois sous le nom de causes finales, et que l'on caractérise mieux en les appelant les harmonies de la nature. Et qu'est-ce qu'arriver à de semblables résultats? C'est pénétrer les desseins de la divinité.» (Guinand 1831:165)

\section{La Terre et l'homme. Leurs rapports}

Le fait d'accorder une importance toute particulière à l'étude des rapports entre la Terre et l'homme est l'un des autres points forts de la pensée d'U. Guinand (cf. GUINAND 1842). Les passages s'y rapportant sont légion. L'homme s'est créé un monde à l'usage de ses besoins. Il est clair pour lui qu'il parvient, par son intelligence, à modifier la nature et à la «sculpter», en partie à sa guise. Les forêts sont défrichées, les fleuves détournés, les marais asséchés. Il est non moins évident que la nature, de son côté, exerce une influence sur l'homme. Et U. Guinand de donner comme exemple, les différences de caractère, d'habitudes, etc., entre l'homme du pôle et celui de l'équateur, entre l'Asiatique et l'Européen, entre l'habitant des montagnes et celui des plaines. Cet auteur attribue notamment ces différences à la variété des climats. Ces derniers, pose-t-il la question, ne déterminent-ils pas, en grande partie, la manière de se nourrir, de se vêtir, de se loger?

Déterministe, la position d'U. Guinand l'est; il ne s'agit toutefois pas d'un déterminisme dogmatique. L'homme, les lignes qui précèdent le démontrent, a la capacité de modeler la nature, de la mettre à son service. Cette capacité de se libérer des contraintes physiques dépend, dit C. RitTer, du degré de civilisation atteint:

"...d'un côté on relève une dépendance inéluctable de l'homme à l'égard de la nature, d'autant plus pressante qu'il est encore proche de l'état d'inconscience et que les peuples vivent en hordes; de l'autre au contraire, une libéralisation toujours plus avancée des peuples civilisés à l'égard des contingences de l'environnement naturel qui perd peu à peu de son emprise.» (RIrTer 1974: 54)

De cette progressive libération de l'homme des contraintes physiques, U. Guinand et C. RITTER en donnent un exemple concret: l'homme et les Alpes. Ecoutons le disciple pour passer, ensuite, au maître. 
«Nier que les Alpes soient une barrière, ce serait méconnaître la vérité; attribuer à cette barrière une valeur absolue, ce serait oublier l'influence énergique de l'homme sur le globe ou s'aveugler dans une exagération romanesque. Les Alpes, sans ressembler pourtant aux fantômes qui s'évanouissent quand on en approche, sont, de nos jours, devenues autant un lien qu'une barrière. Elles ont offert cette même singularité à d'autres époques. L'empire romain se les était assujetties, y avait ouvert des routes et avait transporté ses limites au delà, les entraînant ainsi, avec une partie de la HauteEurope, dans le cercle d'une vie méditerranéenne.» (GuINAND 1838: 62)

Mettons maintenant ces propos avec ceux, de quelques années antérieurs, de C. RITTER:

"C'est ainsi que dans les premiers siècles qui ont suivi la naissance du Christ, le sud civilisé de l'Europe était coupé du nord celtique et germanique non civilisé par une muraille naturelle, à savoir les hautes montagnes impénétrables et impraticables de la chaîne alpine qui traversent l'Europe moyenne d'ouest en est. Au sud des Alpes s'étendaient alors les Etats civilisés de l'Ancien Monde; le versant nord marquait le début du monde barbare. Mais cette forme, une haute paroi dressée par la nature et qui de même que les territoires des peuples était autrefois sa demeure, s'est atténuée au cours de la seconde moitié du dernier millénaire. Au cours du siècle dernier, cette forme qui autrefois faisait barrage s'est faite plus accessible et partout en Europe elle est devenue un pôle d'attraction des peuples, un pays comme les autres. Au cœur même de l'Europe, dans cet ancien désert, toute une série de peuples et d'Etats civilisés ont historiquement proliféré depuis la Provence jusqu'à Steyermark. Les plus profondes vallées évasées, les plus hauts sommets se sont peuplés; le défrichement a fait disparaître les forêts; toutes les vallées et toutes les chaînes de montagnes sont devenues praticables et ont pu être franchies dans presque toutes les directions. La barrière qui à l'époque d'Hannibal et de César séparait le Sud du Nord s'est abaissée et l'on y a vu naître un pays de passage donnant accès à toutes les directions. ...on peut admettre que les Alpes ont vu leurs relations avec leur environnement se modifier, on peut dire que cette forme naturelle grandiose a relâché progressivement son emprise sur l'humanité. Si, dans sa réalité et ses dimensions, la nature physique ne change pour ainsi dire pas, c'est donc bien le facteur historique qui, les progrès de la civilisation aidant, permet aux peuples de se libérer des conditions naturelles que les hommes ont d'ailleurs pu toujours mieux maîtriser, allant même jusqu'à les modifier complètement.» (Ritrer 1974: 141)

Pour U. Guinand, l'homme revêt deux aspects. L'un, universel, immuable, impérissable, se rapporte à l'homme, miroir du Créateur. L'homme est en quelque sorte le reflet des perfections morales de Dieu. L'autre, particulier, variable, fragile, concerne l'homme, fruit de l'éducation et de ses relations avec le globe qu'il habite. L'action de celui-ci est de réagir de diverses manières sur l'élément divin, en appelant l'activité humaine vers un but plutôt que vers un autre. C'est ce qu'U. Guinand appelle «l'influence du climat», déjà évoquée.

\section{Horizontalité, verticalité}

Dans l'œuvre d'U. Guinand apparaît très fréquemment un autre thème cher à $\mathrm{C}$. RITTER. C'est celui de l'étude de la dimension verticale des entités géographiques (continents, pays, etc.). Introduit par U. Guinand à l'Ecole normale de Lausanne dès 1833 , ce thème revêt alors un caractère nouveau, souligné par son directeur:

«Dans ce cours on étudie la terre non-seulement (sic) dans le sens horizontal, mais encore dans le sens vertical. L'étude de la dimension verticale, si négligée des anciens géographes, établit le grand contraste entre les hauts et les bas pays et contribue à faire ressortir la vraie physionomie de tous les membres des continents ainsi que les rapports que ces membres soutiennent entr'eux (sic).» (GAUTHEY 1839: 117)

U. Guinand lui-même souligne cette approche nouvelle de l'étude de l'espace en disant:

«Pour être initiés dans la science du globe, il vous suffira d'étudier avec intelligence les dimensions horizontale et verticale de chaque continent. Certes, il n'y a rien de mystérieux dans tout cela; et, s'il y a quelque chose de surprenant, c'est que tous les géographes du monde, excepté ceux de l'école de RitTer, n'aient pas senti l'importance qu'il y avait à ne négliger aucun de ces deux élémens (sic). C'est, Messieurs, un bien grave reproche à faire à la méthode exclusivement analytique. Chargée, par sa nature et par ses prétentions, de nous révéler entièrement les faits, elle n'est pas même parvenue à remplir convenablement cette tâche; mais elle ne pouvait pas la remplir, parce que les faits ne reçoivent toute leur lumière que de leur coordination naturelle et systématique. Malgré l'éclatant exemple de l'Allemagne, les géographes successeurs de Malte-Brun persistent à ne voir dans les continens (sic), que les dalles juxta-posées (sic) du pavé où marche l'humanité; ils ont cru avoir tout fait en divisant la terre en bassins hydrographiques, et en décrivant successivement le système de chaque fleuve avec ses villes et ses peuples. Mais ce n'est là que la dimension horizontale; je ne dis pas même le développement horizontal, car il faut le demander à la géographie comparée: on ne s'est pas mis en peine de tout ce qui résulte des contrastes entre les hauts et les bas-pays (sic); et de l'influence des premiers sur les seconds; en un mot, on a mesuré des hauteurs, sans prendre garde au développement vertical de la terre.» (GuINAND 1834: 28-29)

U. Guinand veut étudier le globe tel qu'il est, dans toutes ses dimensions, afin de rendre à sa surface son véritable caractère. 


\section{Le triptyque géographique d'U. Guinand}

U. Guinand divise la géographie en trois parties. La géographie physique étudie la Terre soumise aux lois de la nature. La géographie politique, elle, se rapporte à la Terre modifiée par l'homme; l'action de ce dernier est, selon ce géographe, souvent liée à la distribution politique des nations. La géographie astronomique ou géographie mathématique, enfin, envisage la Terre comme élément du système solaire. «Négliger un de ces trois ordres de faits, c'est mutiler la science de la terre.» (Guinand 1831: 167). Arrivé là, ayant évoqué les trois dimensions de la discipline, cet auteur définit cette dernière comme étant la science qui a pour objet la Terre. Mais, contrairement aux enseignements de G. Tobler, collaborateur de H. Pestalozzi (cf. Huber 1997) à Yverdon et de J.-W. HenNing (1812), il aborde une dimension après l'autre. L'étude de la géographie doit commencer, selon lui, par la connaissance du système solaire, par celle des rapports entre ce dernier et la Terre. De cette première approche, il convient de passer à l'étude physique du globe qui, dès lors, trouve sa légitimité. Le globe connu, l'action de l'homme sur la Terre est étudiée. Géographie astronomique, géographie physique et, enfin, géographie politique: tel est l'ordre à suivre selon le Neuchâtelois. Dans ses manuels, nous retrouvons ce plan.

\section{L'œuvre pédagogique d'U. Guinand}

De 1835 à 1880 environ, l'enseignement de la géographie en Suisse romande est donc fortement marqué par l'œuvre d'U. Guinand. Ses manuels, lus par des générations d'écoliers, seront maintes fois réédités (cf. Guinand 1844, 1875, 1880, Huber 1994).

En étudiant de près ses ouvrages, nous devons nous rendre à l'évidence: quoique se réclamant de C. RITTER, le Neuchâtelois est encore tout imprégné de la géographie préritterienne. Il ne suit pas, entre autres, la démarche adoptée par H. Pestalozzi et ses disciples sur les bords du lac de Neuchâtel qui avait tellement séduit C. Ritrer lors de ses visites au grand éducateur. La structure de ses manuels est à l'opposé de la révolutionnaire Explication du plan de Fribourg en Suisse dediée (sic) à la jeunesse de cette ville, pour lui servir de première leçon de géographie, du Père G. GIRARD (1827), premier manuel scolaire de langue française s'inspirant des principes énoncés dans le livre troisième de Emile ou de l'éducation, de J.-J. Rousseau (1969). Il est tout à fait intéressant, à ce propos, de «disséquer» ses ouvrages afin d'étudier leur structure. En effet, la question de l'organisation d'un manuel n'estelle pas révélatrice de la conception qu'a de la discipline son auteur? Dans chacun d'entre eux, l'étude de l'avant-propos, de la préface est, en particulier, de toute importance.
Qu'en est-il, par exemple, de l'épître dédicatoire qui introduit son Esquisse de la terre, suivie d'une description de la Suisse et de celle de la Terre-Sainte? S'adressant explicitement aux instituteurs primaires, il dit:

«Les trois parties, dont il se compose, ne sont pas dans l'ordre où je les ai mises, uniquement par l'impossibilité de se trouver toutes à la fois au commencement, mais parce que, dans un enseignement bien fait, elles doivent se suivre de la même manière que dans cette esquisse. Ainsi, ne faites pas commencer par la Suisse qui est à la fin, ou par les états qui sont au milieu; mais par les océans et les continens (sic), comme le petit livre le fait. Vous vous en trouverez bien à tous égards. Vos enfans (sic) apprendront dès l'entrée ce qu'ils doivent premièrement savoir; quand ils connaîtront bien les océans et les continens (sic), l'étude des états sera un jeu pour eux. La Suisse offrira quelques difficultés de plus que les autres parties; aussi faut-il la réserver pour la dernière.» (GUINAND 1841: III)

Ne sommes-nous pas aux antipodes de la géographie pratiquée à Yverdon quelques années auparavant? La géographie d'U. Guinand est-elle bien celle décrite par L. Vulliemin (1871)? Il n'en est évidemment rien. Ce hiatus entre discours théorique et mise en pratique, chez U. Guinand, suscitera parfois de sévères critiques en Suisse romande. Nous devons entre autres ces dernières au géographe A. Petitpierre (1880).

C'est précisément sur la question, parfaitement matérialisée dans cette épître dédicatoire, de l'opportunité de commencer l'enseignement de la géographie par les divisions mathématiques du globe, par l'«ailleurs» pour aboutir à l'«ici» en d'autres termes, que portent les nombreuses critiques négatives de l'œuvre de cet auteur:

«...à en juger par le manuel de Guinand, l'un des plus répandus chez nous, nous ne sommes pas encore prêts à reconnaître que notre patrie suisse a quelques droits à être connue avant celle des Chinois ou des Hottentots, voire même celle des peuples européens.» (PETITPIERre 1871: 40) Cela dit, certains indices nous incitent à penser qu'U. Guinand ait pu être mal compris, encore de son vivant. Il semble que nombreux aient été les enseignants qui, faute de directives officielles, ont utilisé ses manuels sans préparation. Or, pour d'aucuns, et non des moindres, ces ouvrages ne devaient en aucun cas servir au premier enseignement géographique. L.-F.-F. Gauthey, par exemple, dit:

«Il me paraît indispensable, pour la rendre claire aux jeunes enfants, de la fonder sur l'intuition. Que l'enfant voie donc les choses dans la nature, pour qu'il puisse ensuite bien comprendre leur représentation sur le papier... Ce serait donc après une telle introduction, que j'entamerais l'étude de la géographie générale d'après le plan de l'Esquisse de la Terre.» (GAuThEy 1839: 120)

Dans l'œuvre d'U. Guinand, nous n'avons toutefois trouvé aucune mention d'un éventuel enseignement préparatoire.

Sévère, J. Marti-Henneberg va jusqu'à estimer, à 
propos de L.-F.-F. Gauthey et d'U. Guinand, que «Chacun à son niveau s'efforcera d'éliminer la méthode intuitive.» (MARTI-HENNEBERG 1985: 3). L'état actuel de nos recherches ne nous permet pas, quant à nous, de tenir un language aussi péremptoire.

\section{Un bilan en demi-teintes}

Les propos rassurants de L.-F.-F. Gauthey, de A. et L. Rogivue, de C. Secrétan et de F. Godet quant au rôle d'U. Guinand dans le domaine de la propagation des thèses de C. Rirter en Suisse romande et à l'excellence de son enseignement, ne nous empêchent toutefois pas de penser que, dans la pratique, cet auteur est resté, c'est un euphémisme, bien en-deçà du Père G. GiRARD.

Alors qu'U. Guinand demeure en effet largement dans le domaine des idées, le Père G. Girard, lui, traduit ces dernières dans la pratique pédagogique. Abstraction faite des expériences d'Yverdon, le cordelier fribourgeois reste celui qui, le premier dans le monde francophone, a su interpréter les idées nouvelles et les mettre à la portée des enfants.

\section{Bibliographie}

Gauthey, L.-F.-F. (1839): De l'école normale du canton de Vaud depuis sa fondation en 1833 jusqu'à aujourd'hui. - Lausanne: Ducloux.

GiRARD, G. (1827): Explication du plan de Fribourg en Suisse, dediée (sic) à la jeunesse de cette ville, pour lui servir de première leçon de géographie. - Lucerne: Meyer.

GodET, P. (1913) : Frédéric Godet (1812-1900). - Neuchâtel: Attinger.

Guinand, U. (1831): Précis de géographie comparée par M. Frédéric de Rougemont. - Revue neuchâteloise, avril: 121-126, mai: 163-168, juillet: 217-232.

Guinand, U. (1834): Discours d'ouverture d'un cours de géographie comparée. - Lausanne: Rouiller.

GuiNAND, U. (1838): Discours d'ouverture d'un cours de géographie de la Suisse. - Revue suisse 1:57-74.

Guinand, U. (1841): Esquisse de la Terre, suivie de la description de la Suisse et de celle de la Terre-Sainte. Lausanne: Corbaz et Ducloux.

Guinand, U. (1842): Etude de la Terre, ou éléments de géographie. - Lausanne: Ducloux.

Guinand, U. (1844): Abrégé de géographie, pour les écoles primaires. - Lausanne: Ducloux.

Guinand, U. (1875): Petite esquisse de la Terre suivie de la description de la Suisse et de celle de la TerreSainte. - Lausanne: Bridel.

Guinand, U. (1880): Abrégé de géographie suivi de la description de la Suisse de celle de la Terre-Sainte et de quelques notions de géographie astronomique.Lausanne: Bridel.
Henning, J.-W. (1812): Leitfaden beim methodischen Unterricht in der Geographie. - Iferten: Literarisches Büro.

Huber, B. (1994): Géographie et enseignement primaire: «induction» ou «déduction»? Pérennité d'une question méthodologique. - Thèse de doctorat, Faculté des Sciences économiques et sociales, Université de Genève, Genève.

Huber, B. (1997): Une étape déterminante dans l'évolution de l'enseignement de la géographie: J.-H. Pestalozzi à Yverdon (1805-1825). - Geographica Helvetica 4: $129-132$.

KNAPP, C. (1886): Notice sur les voyageurs et les géographes neuchâtelois lue à la VIe session de l'Association des sociétés suisses de géographie de Genève le 12 août 1886. - Bulletin de la Société neuchâteloise de géographie 2: 65-104.

Marti-Henneberg, J. (1986): L'excursionisme cientific a Catalunya (1876-1900) i la seva contribucio a la geografia i a les ciènces naturals. - Tesi doctoral, Departement de Geografia de la Facultad de Geografia i Historia, Universidad de Barcelona, Barcelona.

Merveilleux, D.-F. DE (1694): La parfaite introduction à la géographie universelle, par une nouvelle méthode abrégée et très facile. - Neuchâtel: Schmid.

OSTERVALD, F.-S. (1773): Géographie élémentaire ou instructions géographiques à l'usage de la jeunesse. Genève: Pellet.

Petitpierre, A. (1871): De l'enseignement de la géographie et de l'histoire. - L'Educateur 3: 39-42, 4: 5560.

Petitpierre, A. (1880): La topographie comme base de l'enseignement géographique. - Le Globe, mémoires: 3-21, 49 - 60.

Ritter, C., traduit par Buret, E. et E. Desor (1836): Géographie générale comparée. - Paris: Paulin.

Ritrer, C., traduit par Nicolas-Obadia, D. (1974): Introduction à la géographie générale comparée. - Paris: Les Belles-Lettres.

RoberT, A. (1909): Les premiers journaux républicains neuchâtelois. - Musée neuchâtelois, juillet/août: 165-186.

Rougemont, F. DE (1831): Précis de géographie comparée. - Neuchâtel: (sans mention d'éditeur).

Rousseau, J.-J. (1969): Emile ou de l'éducation. - Paris: Gallimard.

Schlup, M. (1991): David-François de Merveilleux: un géographe au-dessus de tout soupçon? - Nouvelle revue neuchâteloise 32:29-48.

TAuXE, E. (1922): Ulysse Guinand à Lausanne 1832-1885. - Musée neuchâtelois, mars/avril: 49-67.

VUILlEmin, L. (1871): Souvenirs racontés à ses petitsenfants. - Lausanne: Bridel.

\section{Sources manuscrites}

Anonyme (1833-1834): Procès-verbaux des séances du Comité de l'Ecole normale. 
Charx, P. (1885): Conseils et directions sur les moyens de populariser l'enseignement de la géographie.

Marti-Henneberg, J. (1985): L'enseignement de la géographie et la méthode intuitive dans le canton de Vaud au XIXe siècle.

Rogivue, A., Rogivue, L. \& C. Secrétan (1832): Cours de géographie physique générale rédigé d'après les leçons de Mr. le professeur Guinand.

\section{Résumé: Ulysse Guinand (1810-1885), un géographe suisse romand influent jadis, oublié aujourd'hui}

Le présent article se propose d'évoquer l'œuvre, fort peu connue de nos jours, du géographe neuchâtelois U. Guinand qui, au XIXe siècle, a joui d'une certaine notoriété dans le domaine de l'éducation notamment. Adepte de C. Rirter, il se targuera d'être un pionnier relativement à la diffusion de ses idées en Suisse romande. Après avoir évoqué quelques éléments biographiques afférents à cet auteur, ce texte met en évidence certaines des idées-forces de C. RITTER et les prises de position d'U. Guinand qui s'y rapportent. Sont successivement évoqués l'abîme qui sépare la géographie de tradition française et celle d'obédience germanique, le rôle de la Providence dans l'organisation de la Terre, les rapports entre l'homme et cette dernière, la prise en compte des dimensions horizontale et verticale des entités spatiales, le triptyque géographique d'U. Guinand et, enfin, l'œuvre pédagogique de celui-ci.

\section{Zusammenfassung: Ulysse Guinand (1810-1885), ein bedeutender Geograph der französischen Schweiz, der heute vergessen ist}

Der vorliegende Artikel ruft das Werk des Neuenburger Geographen U. Guinand in Erinnerung, der auf dem Gebiet der Erziehung im 19. Jahrhundert eine bedeutende Rolle gespielt hat. Als C. Ritrers Anhänger hat er dessen Ideen in der französischen Schweiz verbreitet. Der Text enthält zahlreiche biographische Angaben über U. Guinand. Es werden die bedeutenden Ideen von C. Ritrer und die diesbezügliche Stellungnahme U. Guinands aufgezeigt. Grosse Unterschiede zwischen der Geographie französischer Tradi- tion und deutschsprachiger Prägung werden dargelegt, ebenso werden die Rolle der Vorsehung in der Organisation der Erde und die Beziehungen der Menschen zur Erde beleuchtet, wobei den horizontalen und vertikalen räumlichen sowie den politisch-, mathematisch- und physiogeographischen Dimensionen Rechnung getragen wird. Abschliessend wird U. Guinands pädagogisches Werk behandelt.

\section{Summary: Ulysse Guinand (1810-1885), a Swiss French Geographer, Well Known in the 19th Century, Forgotten Today}

This article deals with the work of the geographer U. Guinand, who lived in the 19th century. Although unknown today, this Swiss-French geographer has had an important influence on the scholastic Geography of his region. As C. RitTer's follower, U. Guinand greatly contributed to the diffusion of RITTER's ideas in the French part of Switzerland. This text gives biographical information about the geographer and his ideas. It underlines the difference between French and German Geography at this time. The article also emphasizes U. Guinand's conception regarding the role of Providence in the earth's organisation and the interrelationship between man and his environment. Finally, $\mathrm{U}$. Guinand's role in the teaching of Geography is highlighted.

Dr Bernard Huber, Université de Genève (FAPSE), 9, rte de Drize, $\mathrm{CH}-1227$ Carouge.

e-mail: bernard.huber@pse.unige.ch 